\title{
An improved comprehensive evaluation model and its application
}

\author{
Wenyi Zeng ${ }^{1}$ Shuang Feng ${ }^{2}$ \\ ${ }^{1}$ College of Information Science and Technology, Beijing Normal University, \\ No. 19, Xinjiekouwai Ave., Beijing, 100875, P.R. China \\ E-mail: zengwy@bnu.edu.cn \\ ${ }^{2}$ School of Applied Mathematics, Beijing Normal University, \\ Zhuhai Guangdong, 519087, P.R. China \\ E-mail: fengshuang@mail.bnu.edu.cn
}

Received 3 November 2013

Accepted 29 March 2014

\begin{abstract}
Since comprehensive evaluation model was introduced by Wang in 1984, the comprehensive evaluation model has many successful applications in real life. But some examples show the inefficiency of the comprehensive evaluation model. In order to process these problems, in this paper, we introduce two concepts of standardized fuzzy set and normalized fuzzy set, propose a novel method to improve the comprehensive evaluation model and its algorithm. Finally, we use three numerical examples to illustrate our proposed model reasonable and point out that our proposed method can avoid some inefficiencies in applications.
\end{abstract}

Keywords: Fuzzy set; fuzzy relation; standardized fuzzy set; normalized fuzzy set; comprehensive evaluation matrix

\section{Introduction}

Evaluation method and evaluation model have extensively been applied in many fields such as decision analysis, supporting system and system engineering 1,2 . Generally, decision makers usually face to two kinds of information: one is complete information, the other is incomplete information. Aimed at the complete information, the analytic hierarchy process (AHP) introduced by Saaty ${ }^{3}$ has extensively been applied in economic analysis, technology transfer and population forecast, and has obtained many successful applications. However, considering the incomplete information in real life, it is difficult for people to use AHP with multiplicative preferences. Since fuzzy set theory was introduced by Zadeh ${ }^{4}$, Bellman and Zadeh ${ }^{5}$ began to investi- gate decision-making in fuzzy environment. Fuzzy set plays a significant role in processing and transforming imprecise information effectively and flexibly, thus, decision makers choose fuzzy sets to make some meaning choices due to their different cultural and educational backgrounds, personal habits and vague nature of human judgement $6,7,8$.

Comprehensive evaluation proposed by Wang 9 is an important mathematical model. Since then, it has extensively been applied in many fields such as environmental evaluation ${ }^{10,11}$, weather forecast ${ }^{12}$, teaching evaluation ${ }^{13}$, risk management ${ }^{14,15}$, power generation projects ${ }^{16}$, car retrieval system ${ }^{17}$ and so on. As the comprehensive evaluation model is deeply developed and widely applied, and the lacking of profound insights with respect to the mathe- 
matical fundamentals of the comprehensive evaluation model has also brought along some ambiguous results in real-world applications, comprehensive evaluation model may prevent people from making correct decisions. Some typical examples are reported in ${ }^{8,9}$ to illustrate the inefficiency of the comprehensive evaluation model. In order to avoid this problem, multi-hierarchical comprehensive evaluation model is introduced by some researchers. However, this type of model often results in the increasing amount of computation. Guo et al ${ }^{18}$ reviewed the model and application of comprehensive evaluation.

In this paper, we introduce two concepts of standardized fuzzy set and normalized fuzzy set, propose a novel method to improve the comprehensive evaluation model and use three numerical examples to illustrate our proposed model reasonable.

\section{Preliminaries}

We consider finite universe of discourse set through out this paper. $U=\left\{u_{1}, u_{2}, \cdots, u_{n}\right\}$ denotes the universe of discourse and $\mathscr{F}(U)$ denotes the set of all fuzzy sets on $U$. For a fuzzy set $A \in \mathscr{F}(U)$, it can be represented by

$$
A=A\left(u_{1}\right) / u_{1}+A\left(u_{2}\right) / u_{2}+\cdots+A\left(u_{n}\right) / u_{n}
$$

where $A\left(u_{i}\right)$ is the degree of membership function of fuzzy set $A$ with respect to $u_{i}, i=1,2, \cdots, n$.

Definition $1 \quad h(A)=\bigvee_{i=1}^{n} A\left(u_{i}\right)$ is called the height of the fuzzy set $A$.

Definition $2|A|=\sum_{i=1}^{n} A\left(u_{i}\right)$ is called the cardinality of the fuzzy set $A$.

Definition 3 For $A \in \mathscr{F}(U), \quad S(A)=$ $\frac{A\left(u_{1}\right)}{h(A)} / u_{1}+\frac{A\left(u_{2}\right)}{h(A)} / u_{2}+\cdots+\frac{A\left(u_{n}\right)}{h(A)} / u_{n}$ is called the standardized fuzzy set of the fuzzy set $A$. $N(A)=\frac{A\left(u_{1}\right)}{|A|} / u_{1}+\frac{A\left(u_{2}\right)}{|A|} / u_{2}+\cdots+\frac{A\left(u_{n}\right)}{|A|} / u_{n}$ is called the normalized fuzzy set of the fuzzy set $A$.

$S(A)$ and $N(A)$ are two fuzzy sets transformed from the fuzzy set $A$. We shall employ a simple example to explain them: suppose the fuzzy set $A$ represent the concept "important". Then the membership function of $S(A)$ means the degrees of "importance" relative to the element of $U$ which has the largest degree of "importance". And the membership function of $N(A)$ means the weights of degree of "importance" with respect to every element of $U$ relative to the sum of degrees of membership function of the fuzzy set $A$.

Definition 4 A mapping $T:[0,1] \times[0,1] \rightarrow$ $[0,1]$ is called a quasi-triangular norm if it satisfies the following properties:

(1) $T(0,0)=0, T(1,1)=1$;

(2) $T(x, y)=T(y, x), \forall x, y \in[0,1]$;

(3) $\left(x \leqslant x^{\prime}, y \leqslant y^{\prime}\right) \Rightarrow T(x, y) \leqslant T\left(x^{\prime}, y^{\prime}\right)$;

(4) $T(T(x, y), z)=T(x, T(y, z)), \forall x, y, z \in[0,1]$.

A quasi-triangular norm $T$ is called a triangular norm if it satisfies

$$
\left(1^{\prime}\right) T(x, 1)=x
$$

and $T$ is called a complementary triangular norm if it satisfies

$$
\left(1^{\prime \prime}\right) T(x, 0)=x
$$

Note that either $\left(1^{\prime}\right)$ or $\left(1^{\prime \prime}\right)$ will imply $(1)$.

When a quasi-triangular(triangular, or complementary triangular) norm is a continuous function, we call it a continuous quasi-triangular(triangular, or complementary triangular) norm.

Definition 5 Let $T$ be a triangular norm and $T^{*}$ be a complementary triangular norm, respectively, then $T$ and $T^{*}$ are called correlated if they satisfy

$$
\text { (5) } T(x, y)+T^{*}(1-x, 1-y)=1
$$

Theorem 1 For any triangular norm $T$, there exists a unique complementary triangular norm $T^{*}$ that correlates with $T$; if $T$ is continuous, so is $T^{*}$. Conversely, for any complementary triangular norm $T^{*}$, there exists a unique triangular norm $T$ that correlates with $T^{*}$; if $T^{*}$ is continuous, so is $T$.

Proof We will prove the "existence" since the "uniqueness" and the "continuity" are trivial.

Form the condition (5), for any triangular norm $T$ we can get

$$
T^{*}(x, y)=1-T(1-x, 1-y)
$$

Clearly, this is a mapping from $[0,1] \times[0,1]$ to $[0,1]$. 
We now prove $T^{*}$ satisfies $\left(1^{\prime \prime}\right),(2),(3)$ and (4).

(1") $T^{*}(x, 0)=1-T(1-x, 1-0)=1-T(1-$ $x, 1)=1-(1-x)=x$.

(2) $T^{*}(x, y)=1-T(1-x, 1-y)=1-T(1-$ $y, 1-x)=T^{*}(y, x)$.

(3) If $x \leqslant x^{\prime}$ and $y \leqslant y^{\prime}$, then

$$
\begin{aligned}
T^{*}(x, y) & =1-T(1-x, 1-y) \\
& \leqslant 1-T\left(1-x^{\prime}, 1-y^{\prime}\right) \\
& =T^{*}(x, y)
\end{aligned}
$$

(4)

$$
\begin{aligned}
T^{*}\left(T^{*}(x, y), z\right) & =1-T\left(1-T^{*}(x, y), 1-z\right) \\
& =1-T(T(1-x, 1-y), 1-z) \\
& =1-T(1-x, T(1-y, 1-z)) \\
& =1-T\left(1-x, 1-T^{*}(y, z)\right) \\
& =T^{*}\left(x, T^{*}(y, z)\right)
\end{aligned}
$$

So $T^{*}$ is a complementary triangular norm and it correlates with $T$.

Similarly, we can prove that $T$ correlates with $T^{*}$ satisfies $\left(1^{\prime}\right),(2),(3)$ and (4) when $T^{*}$ is complementary triangular norm.

Hence, we complete the proof of Theorem 1.

Thus, The following are examples of triangular norms from $[0,1]$ to $[0,1]$.

$\bigwedge:(x, y) \rightarrow \bigwedge(x, y)=x \wedge y=\min (x, y) ;$

$\bullet:(x, y) \rightarrow \bullet(x, y)=x \bullet y=x y$;

$\odot:(x, y) \rightarrow \odot(x, y)=x \odot y=\max (x+y-1,0)$.

The following are examples of complementary triangular norms from $[0,1]$ to $[0,1]$.

$\bigvee:(x, y) \rightarrow \bigvee(x, y)=x \vee y=\max (x, y)$;

$+:(x, y) \rightarrow+(x, y)=x+y=x+y-x y, ;$

$\oplus:(x, y) \rightarrow \oplus(x, y)=x \oplus y=\min (x+y, 1)$.

And we can get the following conclusion.

$$
(\bigwedge)^{*}=\bigwedge, \quad \bullet^{*}=\dot{+}, \quad \odot^{*}=\oplus
$$

\section{Mathematical model of comprehensive evaluation}

Suppose the factor set $U=\left\{u_{1}, u_{2}, \cdots, u_{n}\right\}$ and the evaluation set $V=\left\{v_{1}, v_{2}, \cdots, v_{m}\right\}$, then the fuzzy relation between $U$ and $V$ can be represented by a evaluation matrix $R$ denoted by

$$
R=\left(\begin{array}{cccc}
r_{11} & r_{12} & \cdots & r_{1 m} \\
r_{21} & r_{22} & \cdots & r_{2 m} \\
\vdots & \vdots & & \vdots \\
r_{n 1} & r_{n 2} & \cdots & r_{n m}
\end{array}\right)
$$

where $r_{i j}$ represents the evaluation value of the $i$ th factor $u_{i}$ about the $j$ th evaluation $v_{j}$.

Weight is a preference relation and displays the importance of every factor, thus, it is a fuzzy set on factor set $U$ since people do not acquire the exact definition and clear extension for the concept of "importance". Denote $A=\left(a_{1}, a_{2}, \cdots, a_{n}\right) \in \mathscr{F}(U)$, then $B=A \circ R=\left(b_{1}, b_{2}, \cdots, b_{m}\right) \in \mathscr{F}(V)$, where "o" denotes the composition operator of fuzzy relation. Based on the composition operation of fuzzy relation, Wang ${ }^{9}$ proposed the following mathematical model for comprehensive evaluation.

Model I $M(\bigwedge, \bigvee)$ and $b_{j}=\bigvee_{i=1}^{n}\left(a_{i} \wedge r_{i j}\right)$.

In the following, we give some applications for the comprehensive evaluation model.

Example 1 Cloth selection.

Assume the basic factors of interest in the cloth selection consist of $u_{1}=$ "style", $u_{2}=$ "quality", $u_{3}=$ "price", i.e., $U=\left\{u_{1}, u_{2}, u_{3}\right\}$. The verbal grades used for the selection are $v_{1}=$ "best", $v_{2}=$ "good", $v_{3}=$ "fair", $v_{4}=$ "poor", i.e., $V=$ $\left\{v_{1}, v_{2}, v_{3}, v_{4}\right\}$. For a particular piece of cloth $u$, the single-factor evaluation may be carried out by professionals or customers. For instance, if the survey results on the "style" factor are 0.7 for the "best", 0.2 for the "good", 0.1 for the "fair", and none for the "poor", then the single-factor evaluation vector $r_{1}$ is

$$
r_{1}=(0.7,0.2,0.1,0)
$$

Similarly, we can obtain the following singlefactor evaluation vectors for $u_{2}$ and $u_{3}$ :

$$
r_{2}=(0.2,0.4,0.3,0.1)
$$

and

$$
r_{3}=(0.1,0.3,0.4,0.2)
$$

Now we have the following evaluation matrix $R$ : 


$$
R=\left(\begin{array}{cccc}
0.7 & 0.2 & 0.1 & 0 \\
0.2 & 0.4 & 0.3 & 0.1 \\
0.1 & 0.3 & 0.4 & 0.2
\end{array}\right)
$$

If a customer's weight vector with respect to the three factors is $A=(0.5,0.3,0.2)$, then by applying the above-mentioned mathematical model of comprehensive evaluation, we can compute the comprehensive evaluation vector as follows.

$$
\begin{aligned}
B & =A \circ R \\
& =(0.5,0.3,0.2) \circ\left(\begin{array}{cccc}
0.7 & 0.2 & 0.1 & 0 \\
0.2 & 0.4 & 0.3 & 0.1 \\
0.1 & 0.3 & 0.4 & 0.2
\end{array}\right) \\
& =(0.5,0.3,0.3,0.2)
\end{aligned}
$$

Because the largest component of the fuzzy set $B$ is $B\left(v_{1}\right)=0.5$, this piece of cloth received the "best" rating from the customers.

Example 2 Teaching evaluation.

Assume the basic factors that influence students' teaching evaluation are $u_{1}=$ "clarity and understanding", $u_{2}=$ "proficiency in teaching material", $u_{3}=$ "liveliness and stimulation", and $u_{4}=$ "writing neatness", i.e., $U=$ $\left\{u_{1}, u_{2}, u_{3}, u_{4}\right\}$. The verbal grades used for the selection are $v_{1}=$ "excellent", $v_{2}=$ "very good", $v_{3}=$ "good", $v_{4}=$ "poor", i.e., $V=\left\{v_{1}, v_{2}, v_{3}, v_{4}\right\}$. For a particular teacher $u$, by selecting an appropriate group of students and faculty, we can have them respond with their ratings on each factor and then obtain the single-factor evaluation on each factor. As in the previous example, we have the following evaluation matrix $R$ :

$$
R=\left(\begin{array}{cccc}
0.4 & 0.5 & 0.1 & 0 \\
0.6 & 0.3 & 0.1 & 0 \\
0.1 & 0.2 & 0.6 & 0.1 \\
0.1 & 0.2 & 0.5 & 0.2
\end{array}\right)
$$

For a specific weight vector $A=$ $(0.5,0.2,0.2,0.1)$, and applying the comprehensive evaluation model $M(\bigvee, \wedge)$, it is easy to find

$$
B=A \circ R=(0.4,0.5,0.2,0.1)
$$

From our results, we conclude that teacher $u$ should be rated as "very good".

Example 3 Let factor set be $U=$ $\left\{u_{1}, u_{2}, u_{3}, u_{4}, u_{5}, u_{6}, u_{7}, u_{8}, u_{9}, u_{10}\right\}$, and evaluation set $V=\left\{v_{1}, v_{2}, v_{3}, v_{4}\right\}$, if we have the weight vector $A$ and the fuzzy relation $R$ as follows.

$$
\begin{gathered}
A=(0.2,0.15,0.1,0.05,0.07,0.03 \\
0.12,0.08,0.09,0.11)
\end{gathered}
$$

and

$$
R=\left(\begin{array}{cccc}
0.4 & 0.3 & 0.2 & 0.1 \\
0.5 & 0.3 & 0.2 & 0 \\
0.3 & 0.3 & 0.3 & 0.1 \\
0.2 & 0.2 & 0.4 & 0.2 \\
0.6 & 0.4 & 0 & 0 \\
0.2 & 0.3 & 0.5 & 0 \\
0.5 & 0.1 & 0.3 & 0.1 \\
0.2 & 0.3 & 0.4 & 0.1 \\
0.5 & 0.3 & 0.1 & 0.1 \\
0.4 & 0.2 & 0.3 & 0.1
\end{array}\right)
$$

Then we can compute the comprehensive evaluation vector as follows.

$$
B=A \circ R=(0.2,0.2,0.2,0.1)
$$

This does not permit us to draw conclusions.

The comprehensive evaluation model can be also used as a simple kind of fuzzy control model by many scholars, the weight vector $A$ can be viewed as a fuzzy input in $\mathscr{F}(U)$, the fuzzy relation $R$ acts as a transformer that turns the given "input", the fuzzy set $A$, into an "output", the fuzzy set $B$. Therefore, when the input vectors are basically proportional, thus, people have sufficient reason to believe that the output vectors should almost be the proportional. But we sometimes can not conclude the result that we need here.

Example 4 Suppose the fuzzy relation $R$ as follows.

$$
R=\left(\begin{array}{ccccc}
0 & 0.04 & 0.88 & 0.32 & 0 \\
0 & 0.73 & 0.60 & 0.16 & 0.06 \\
0 & 0.47 & 0.73 & 0.19 & 0.07 \\
0 & 0 & 0.78 & 0.57 & 0.15
\end{array}\right)
$$


For two kinds of weight vectors,

$$
A_{1}=(0.8,0.6,0.4,0.4)
$$

and

$$
A_{2}=(0.4,0.3,0.15,0.15)
$$

Then we apply model $M(\wedge, \bigvee)$ and obtain

$$
\begin{gathered}
B_{1}=A_{1} \circ R=(0,0.6,0.8,0.4,0.15) \\
B_{2}=A_{2} \circ R=(0,0.3,0.4,0.32,0.15)
\end{gathered}
$$

Obviously, for the comprehensive evaluation model, the input vectors are almost proportional, but the output vectors have a large difference.

Based on the above-mentioned questions, researchers have two kinds of methods to process them. First, aimed at the large number of factors, it is common to group factors into categories according to some attributes or properties for the group factors. For example, in rank ordering the best ten business schools in the U.S., one may consider such factors as: teaching, research, program design, employment prospects, etc. Obviously we have a weight distribution among these factors, then every factor will get a very small weight value because of the large number of factors, thus, it is possible that we could not get the correct conclusion when we proceed with the comprehensive evaluation model. Hence, we have to integrate some small factors to form a large factor. For example, factors that influence teaching evaluation have many such as quality of faculty, quality of students, teaching facilities, and so on, then we can integrate these small factors to form a large factor "teaching". Thus, some researchers have proposed the multi-hierarchical comprehensive evaluation model. Second, people introduced the generalized fuzzy "AND" operator " $*$ " and the generalized fuzzy "OR" operator " “*” into the comprehensive evaluation model, thus we have the generalized comprehensive evaluation model, denoted by $M\left(\dot{*}^{*} *\right)$, where $B=\left(b_{1}, b_{2}, \cdots, b_{m}\right)$ and $b_{j}=$ $\left(a_{1} \dot{*} r_{1 j}\right) *\left(a_{2} \dot{*} r_{2 j}\right) * \cdots *\left(a_{n} \dot{*} r_{n j}\right), j=1,2, \cdots, m$. Therefore, some researchers have proposed the following comprehensive evaluation models.
Model II $M(\cdot, \bigvee)$ and $b_{j}=\bigvee_{i=1}^{n}\left(a_{i} \cdot r_{i j}\right), j=$ $1,2, \cdots, m$.

Model III $M(\cdot, \oplus)$ and $b_{j}=\bigoplus_{i=1}^{n}\left(a_{i} \cdot r_{i j}\right)=$ $\sum_{i=1}^{n}\left(a_{i} \cdot r_{i j}\right), j=1,2, \cdots, m$.

Model IV $M(\bigwedge, \oplus)$ and $b_{j}=\bigoplus_{i=1}^{n}\left(a_{i} \bigwedge r_{i j}\right)=$ $\min \left(1, \sum_{i=1}^{n}\left(a_{i} \wedge r_{i j}\right)\right), j=1,2, \cdots, m$.

Model V $M($ power,$\Lambda)$ and $b_{j}=\bigwedge_{i=1}^{n} r_{i j}^{a_{i}}, j=$ $1,2, \cdots, m$.

We find that the operator, " $*$ "="power", does not satisfy the associative law. Therefore Model $\mathrm{V}$ can not be applied in comprehensive evaluation. And so far as the decision makers are concerned, they expect to achieve a consistent conclusion no matter what method is employed: single-layered model or multi-hierarchical model, i.e., the thoughts of decision makers should be stable aimed at the all situations. Furthermore, we have the following theorem.

Theorem 2 If a comprehensive evaluation model satisfies the requirement of stability of decision-making thinking, then the generalized fuzzy operators should satisfy the distributive law. Namely,

$$
s \dot{*}\left(\stackrel{+}{*}_{i=1}^{n} t\right)=\stackrel{+}{*}_{i=1}^{n}(s \dot{*} t), \quad s, t \in[0,1]
$$

Proof For the convenience, we only consider 2-layered comprehensive evaluation model. Let $U=\left\{u_{1}, u_{2}, \cdots, u_{m}\right\}$ be the factor set, where $u_{i}=$ $\left\{u_{i_{1}}, u_{i_{2}}, \cdots, u_{i_{n_{i}}}\right\}$. Let the evaluation set $V=$ $\left\{v_{1}, v_{2}, \cdots, v_{n}\right\}$. Assume that $A=\left(a_{1}, a_{2}, \cdots, a_{m}\right)$ is the set of weights with respect to factor set $U$ and $A_{i}=\left(a_{i_{1}}, a_{i_{2}}, \cdots, a_{i_{n_{i}}}\right)$ is the set of weights with respect to the factor $u_{i}(i=1,2, \cdots, m)$. If multi-hierarchical comprehensive evaluation model is adopted, for every $i=1,2, \cdots, m$, we have 


$$
\begin{aligned}
& B_{i}=\left(a_{i_{1}}, a_{i_{2}}, \cdots, a_{i_{n_{i}}}\right) \circ \\
& \left(\begin{array}{cccc}
r_{i_{1}, 1} & r_{i_{1}, 2} & \cdots & r_{i_{1}, n} \\
r_{i_{2}, 1} & r_{i_{2}, 2} & \cdots & r_{i_{2}, n} \\
\vdots & \vdots & & \vdots \\
r_{i_{n_{i}}, 1} & r_{i_{n_{i}}, 2} & \cdots & r_{i_{n_{i}}, n}
\end{array}\right) \\
& =\left(*_{j=1}^{+{ }^{n_{i}}}\left(a_{t_{i}} \dot{n_{i}} r_{i_{j}, 1}\right), *_{j=1}^{+^{n_{i}}}\left(a_{i_{j}} \dot{*} r_{i_{j}, 2}\right), \cdots,\right. \\
& \left.*_{j=1}\left(a_{i_{j}} * r_{i_{j}, n}\right)\right) \\
& B=\left(a_{1}, a_{2}, \cdots, a_{m}\right) \circ\left(\begin{array}{c}
B_{1} \\
B_{2} \\
\vdots \\
B_{m}
\end{array}\right)
\end{aligned}
$$

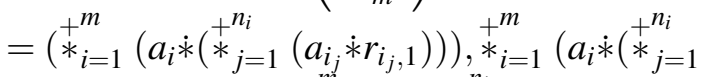

$$
\begin{aligned}
& \left.\left.\left.\left(a_{i_{j}} \dot{*} r_{i_{j}, 2}\right)\right)\right), \cdots, *_{i=1}^{+m}\left(a_{i} \dot{*}\left(*^{+_{i}}{ }_{j=1}^{*_{i}}\left(a_{i_{j}} \dot{*} r_{i_{j}, n}\right)\right)\right)\right)
\end{aligned}
$$

Now consider the single-layered comprehensive decision-making model, the factor set $U^{\prime}=$ $\left\{u_{11}, u_{12}, \cdots, u_{1 n_{1}}, u_{21}, u_{22}, \cdots, u_{2 n_{2}}, \cdots, u_{m 1}, u_{m 2}, \cdots\right.$, $\left.u_{m n_{m}}\right\}$, and the set of weights $A^{\prime}=\left(a_{1} \dot{*} a_{11}, a_{1} \dot{*} a_{12}\right.$,

$\cdots, a_{1} \dot{*} a_{1 n_{1}}, a_{2} \dot{*} a_{21}, a_{2} \dot{*} a_{22}, \cdots, a_{2} \dot{*} a_{2 n_{2}}, \cdots, a_{m} \dot{*} a_{m 1}$, $\left.\cdots, a_{m} \dot{*} a_{m n_{m}}\right)$. Then

$$
\begin{aligned}
& B^{\prime}=A^{\prime} \circ R^{\prime}=\left(a_{1} \dot{*} a_{11}, a_{1} \dot{*} a_{12}, \cdots, a_{m} \dot{*} a_{m n_{m}}\right) \circ \\
& \left(\begin{array}{cccc}
r_{11,1} & r_{11,2} & \cdots & r_{11, n} \\
r_{12,1} & r_{12,2} & \cdots & r_{12, n} \\
\vdots & \vdots & & \vdots \\
r_{m n_{m}, 1} & r_{m n_{m}, 2} & \cdots & r_{m n_{m}, n}
\end{array}\right) \\
& =\left(*_{i=1}^{+m}\left(*_{j=1}^{*^{n_{i}}}\left(a_{i} \dot{*} a_{i_{j}} \dot{*} r_{i_{j}, 1}\right)\right), *_{i=1}^{+^{m}}\left(* _ { j = 1 } ^ { + ^ { n _ { i } } } \left(a_{i} \dot{*} a_{i_{j}} \dot{*}\right.\right.\right. \\
& \left.\left.\left.\cdots, r_{i j, 2}\right)\right), *_{i=1}^{+m}\left(*_{j=1}^{n_{i}}\left(a_{i} \dot{*} a_{i_{j}} \dot{*} r_{i_{j}, n}\right)\right)\right)
\end{aligned}
$$

According to the stability of decision-making thinking, we have $B=B^{\prime}$, then

$\stackrel{+}{*}_{i=1}^{m}\left(a_{i} \dot{*}\left(\stackrel{+}{*}_{j=1}^{n_{i}}\left(a_{i_{j}} \dot{*} r_{i_{j}, k}\right)\right)\right)=\stackrel{+}{*}_{i=1}^{m}\left({ }^{+}{ }_{j=1}^{n_{i}}\left(a_{i} \dot{*} a_{i_{j}} \dot{*} r_{i_{j}, k}\right)\right)$

for every $k=1,2, \cdots, n$.

Thus

$$
a_{i} \dot{*}\left({ }^{+n_{i}}{ }_{j=1}^{n_{j}}\left(a_{i_{j}} \dot{*} r_{i_{j}, k}\right)\right)={\stackrel{+}{*_{j}}}_{j=1}^{n_{i}}\left(a_{i} \dot{*} a_{i_{j}} \dot{*} r_{i_{j}, k}\right)
$$

which implies that the operators “*** and “*” satisfy the distributive law.
Hence, we complete the proof of Theorem 2.

However, when “* $* \otimes$ " and “* $=$ $\wedge "$ we have $0.4=0.4 \wedge(0.5 \oplus 0.3) \neq$ $(0.4 \wedge 0.5) \oplus(0.4 \wedge 0.3)=0.7$. Therefore, Model IV dees not satisfy the distributive law, so it can not be applied in comprehensive evaluation. And it has been pointed out by Professor Wang ${ }^{9}$ that there is no rational result from Model IV.

\section{Improvement of comprehensive evaluation model}

Now we have three models left after selection: $M(\bigwedge, \bigvee), M(\cdot, \bigvee)$ and $M(\cdot, \oplus)$. And it will lead to inefficiency of using the comprehensive evaluation model if the actual meaning of the three models is not well understood. Therefore, we propose an improvement method, namely, standardizing or normalizing the weight vector $A$ and the fuzzy relation $R$, thus, we have the fuzzy set $S(A)$ or $N(A)$ and the fuzzy relation $S(R)$ or $N(R)$.

1. For Model $M(\bigwedge, \bigvee)$, apply standardization and normalization to the fuzzy set $A$ and the fuzzy relation $R$, respectively, then we have $A^{\prime}=S(A)$ and $R^{\prime}=N(R)$. Thus, the model output $B=A^{\prime} \circ R^{\prime}$ where $b_{j}=\bigvee_{i=1}^{n}\left(a_{i}^{\prime} \bigwedge r_{i j}^{\prime}\right), j=1,2, \cdots, m$.

2. For Model $M(\cdot, \bigvee)$, apply standardization and normalization to the fuzzy set $A$ and the fuzzy relation $R$, respectively, then we have $A^{\prime}=S(A)$ and $R^{\prime}=N(R)$. Thus, the model output $B=A^{\prime} \circ R^{\prime}$ where $b_{j}=\bigvee_{i=1}^{n}\left(a_{i}^{\prime} \cdot r_{i j}^{\prime}\right), j=1,2, \cdots, m$.

3. For Model $M(\cdot, \oplus)$, apply normalization to the fuzzy set $A$ and the fuzzy relation $R$, respectively, then we have $A^{\prime}=N(A)$ and $R^{\prime}=N(R)$. Thus, the model output $B=A^{\prime} \circ R^{\prime}$ where $b_{j}=\sum_{i=1}^{n}\left(a_{i}^{\prime} \cdot r_{i j}^{\prime}\right), j=$ $1,2, \cdots, m$.

Further, we introduce the multifactorial function to investigate the comprehensive evaluation model. Let $[0,1]^{m}$ be an m-dimensional cube and $X=$ $\left(x_{1}, x_{2}, \cdots, x_{m}\right)$ and $Y=\left(y_{1}, y_{2}, \cdots, y_{m}\right) \in[0,1]^{m}$, we define $X \leqslant Y$ if and only if $(\forall j)\left(x_{j} \leqslant y_{j}\right)$, then " $\leqslant$ " is a partial relation on the cube. 
Definition 6 A mapping $M_{m}:[0,1]^{m} \rightarrow[0,1]$ is called an additive standard multifactorial function if it satisfies the following properties:

1) $X \leqslant Y \Rightarrow M_{m}(X) \leqslant M_{m}(Y)$;

2) $\bigwedge_{j=1}^{m} x_{j} \leqslant M_{m}(X) \leqslant \bigvee_{j=1}^{m} x_{j}$

3) $M_{m}\left(x_{1}, x_{2}, \cdots, x_{m}\right)$ is a continuous function for each variable $x_{j}$.

We call this kind of function an $A S M_{m}-f u n c$, and the set of all $A S M_{m}-$ funcs is denoted by $\mathscr{M}_{m}$. Specially, $A S M_{1}-$ func is an identity mapping from $[0,1]$ to $[0,1]$.

The following are examples of $A S M_{m}$ - funcs from $[0,1]^{m}$ to $[0,1]$.

(a) $f_{1}:\left(x_{1}, x_{2}, \cdots, x_{m}\right) \rightarrow f_{1}\left(x_{1}, x_{2}, \cdots, x_{m}\right)=$ $\bigwedge_{j=1}^{m} x_{j}$

$j=1$

(b) $f_{2}:\left(x_{1}, x_{2}, \cdots, x_{m}\right) \rightarrow f_{2}\left(x_{1}, x_{2}, \cdots, x_{m}\right)=$ $\bigvee_{j=1}^{m} x_{j}$

$j=1$

(c) $f_{3}:\left(x_{1}, x_{2}, \cdots, x_{m}\right) \rightarrow f_{3}\left(x_{1}, x_{2}, \cdots, x_{m}\right)=$ $\sum_{j=1}^{m} a_{j} x_{j}$, where $a_{j} \in[0,1]$ and $\sum_{j=1}^{m} a_{j}=1$;

(d) $f_{4}:\left(x_{1}, x_{2}, \cdots, x_{m}\right) \rightarrow f_{4}\left(x_{1}, x_{2}, \cdots, x_{m}\right)=$ $\sum_{j=1}^{m} a_{j}\left(x_{j}\right) x_{j}$, where $a_{j}:[0,1] \rightarrow[0,1], x_{j} \rightarrow a_{j}\left(x_{j}\right)$

is a continuous function and satisfies $\sum_{j=1}^{m} a_{j}\left(x_{j}\right)=1$;

(e) $f_{5}:\left(x_{1}, x_{2}, \cdots, x_{m}\right) \rightarrow f_{5}\left(x_{1}, x_{2}, \cdots, x_{m}\right)=$ $\bigvee_{j=1}^{m}\left(a_{j} x_{j}\right)$, where $a_{j} \in[0,1]$ and $\bigvee_{j=1}^{m} a_{j}=1$;

(f) $f_{6}:\left(x_{1}, x_{2}, \cdots, x_{m}\right) \rightarrow f_{6}\left(x_{1}, x_{2}, \cdots, x_{m}\right)=$ $\bigvee_{j=1}^{m}\left(a_{j} \wedge x_{j}\right)$, where $a_{j} \in[0,1]$ and $\bigvee_{j=1}^{m} a_{j}=1$;


$\left(\prod_{j=1}^{m} x_{j}\right)^{\frac{1}{m}}$;

(h) $f_{8}:\left(x_{1}, x_{2}, \cdots, x_{m}\right) \rightarrow f_{8}\left(x_{1}, x_{2}, \cdots, x_{m}\right)=$ $\left(\frac{1}{m} \sum_{j=1}^{m} x_{j}^{p}\right)^{\frac{1}{p}}, p>0$

(i) $f_{9}:\left(x_{1}, x_{2}, \cdots, x_{m}\right) \rightarrow f_{9}\left(x_{1}, x_{2}, \cdots, x_{m}\right)=$ $\left(\sum_{j=1}^{m} a_{j} x_{j}^{p}\right)^{\frac{1}{p}}, p>0$, where $a_{j} \in[0,1]$ and $\sum_{j=1}^{m} a_{j}=1$.

Theorem 3 Multifactorial function $M_{m}$ satisfies the following properties:

1) $M_{m}(1,1, \cdots, 1)=1$

2) $M_{m}(0,0, \cdots, 0)=0$.

The proof is obvious.

Therefore, for the factor set $U=\left\{u_{1}, u_{2}, \cdots, u_{n}\right\}$ and the evaluation set $V=\left\{v_{1}, v_{2}, \cdots, v_{m}\right\}$. First, we define a one-factor evaluation mapping $\psi$ from $U$ to $\mathscr{F}(V)$ as follows:

$$
u_{i} \rightarrow \psi\left(u_{i}\right)=\left(r_{i 1}, r_{i 2}, \cdots, r_{i m}\right)
$$

Then we have the fuzzy relation between $U$ and $V$, and form an evaluation matrix $R$ denoted by

$$
R=\left(\begin{array}{cccc}
r_{11} & r_{12} & \cdots & r_{1 m} \\
r_{21} & r_{22} & \cdots & r_{2 m} \\
\vdots & \vdots & & \vdots \\
r_{n 1} & r_{n 2} & \cdots & r_{n m}
\end{array}\right)
$$

Now, we take a suitable $A S M_{n}-$ func $M_{n} \in \mathscr{M}_{n}$ and make the multifactorial evaluation:

$$
\begin{aligned}
& B=\left(b_{1}, b_{2}, \cdots, b_{m}\right) \\
& =\left(M_{n}\left(r_{11}, r_{21}, \cdots, r_{n 1}\right), M_{n}\left(r_{12}, r_{22}, \cdots, r_{n 2}\right), \cdots,\right. \\
& \left.M_{n}\left(r_{1 m}, r_{2 m}, \cdots, r_{n m}\right)\right)
\end{aligned}
$$

Clearly, $B \in \mathscr{F}(V)$. If there exists an index $k_{0} \in\{1,2, \cdots, m\}$ such that

$$
b_{k_{0}}=\max \left\{b_{1}, b_{2}, \cdots, b_{m}\right\}
$$

then we should adopt the evaluation $b_{k_{0}}$.

\section{Numerical examples}

In this section, we apply the three improved comprehensive evaluation models to the above-mentioned examples where the inefficiency of traditional model is encountered.

Now we reconsider the example 3 above-mentioned. Let factor set be $U=$ $\left\{u_{1}, u_{2}, u_{3}, u_{4}, u_{5}, u_{6}, u_{7}, u_{8}, u_{9}, u_{10}\right\}$, and the evaluation set $V=\left\{v_{1}, v_{2}, v_{3}, v_{4}\right\}$, if we have

$$
A=(0.2,0.15,0.1,0.05,0.07,0.03 \text {, }
$$

$0.12,0.08,0.09,0.11)$ 
and

$$
R=\left(\begin{array}{cccc}
0.4 & 0.3 & 0.2 & 0.1 \\
0.5 & 0.3 & 0.2 & 0 \\
0.3 & 0.3 & 0.3 & 0.1 \\
0.2 & 0.2 & 0.4 & 0.2 \\
0.6 & 0.4 & 0 & 0 \\
0.2 & 0.3 & 0.5 & 0 \\
0.5 & 0.1 & 0.3 & 0.1 \\
0.2 & 0.3 & 0.4 & 0.1 \\
0.5 & 0.3 & 0.1 & 0.1 \\
0.4 & 0.2 & 0.3 & 0.1
\end{array}\right)
$$

Because $R=N(R)$, thus we apply the model $M(\bigwedge, \bigvee)$ and obtain

$$
\begin{aligned}
A^{\prime} & =S(A) \\
& =(1,0.75,0.5,0.25,0.35,0.15,0.6, \\
& =0.4,0.45,0.55) \\
B^{\prime} & =A^{\prime} \circ R=(0.5,0.35,0.4,0.2)
\end{aligned}
$$

Apply the model $M(\cdot, \bigvee)$ and we obtain

$$
\begin{aligned}
A^{\prime}= & S(A) \\
= & (1,0.75,0.5,0.25,0.35,0.15, \\
& 0.6,0.4,0.45,0.55) \\
B^{\prime}= & A^{\prime} \circ R=(0.4,0.3,0.2,0.1)
\end{aligned}
$$

Apply the model $M(\cdot, \oplus)$ and we obtain

$$
\begin{aligned}
A^{\prime}= & N(A)=A \\
= & (0.2,0.15,0.1,0.05,0.07,0.03 \\
& 0.12,0.08,0.09,0.11) \\
B^{\prime}= & A \circ R=(0.408,0.267,0.245,0.08)
\end{aligned}
$$

Here, we can give the best choice which is the same as and apply the result in real life.

Now we reconsider the example 4 abovementioned. Suppose

$$
R=\left(\begin{array}{ccccc}
0 & 0.04 & 0.88 & 0.32 & 0 \\
0 & 0.73 & 0.60 & 0.16 & 0.06 \\
0 & 0.47 & 0.73 & 0.19 & 0.07 \\
0 & 0 & 0.78 & 0.57 & 0.15
\end{array}\right)
$$

For two kinds of weight vectors,

$$
A_{1}=(0.8,0.6,0.4,0.4)
$$

and

$$
A_{2}=(0.4,0.3,0.15,0.15)
$$

Then, we have:

$$
R^{\prime}=N(R)=\left(\begin{array}{ccccc}
0 & 0.03 & 0.71 & 0.26 & 0 \\
0 & 0.47 & 0.39 & 0.1 & 0.04 \\
0 & 0.32 & 0.5 & 0.13 & 0.05 \\
0 & 0 & 0.52 & 0.38 & 0.1
\end{array}\right)
$$

Apply the model $M(\bigwedge, \bigvee)$ and we obtain

$$
\begin{gathered}
A_{1}^{\prime}=S\left(A_{1}\right)=(1,0.75,0.5,0.5) \\
B_{1}^{\prime}=A_{1}^{\prime} \circ R^{\prime}=(0,0.47,0.71,0.38,0.1) \\
A_{2}^{\prime}=S\left(A_{2}\right)=(1,0.75,0.375,0.375) \\
B_{2}^{\prime}=A_{2}^{\prime} \circ R^{\prime}=(0,0.47,0.71,0.375,0.1)
\end{gathered}
$$

Apply the model $M(\cdot, \bigvee)$ and we obtain

$$
\begin{gathered}
A_{1}^{\prime}=S\left(A_{1}\right)=(1,0.75,0.5,0.5) \\
B_{1}^{\prime}=A_{1}^{\prime} \circ R^{\prime}=(0,0.3525,0.71,0.26,0.05) \\
A_{2}^{\prime}=S\left(A_{2}\right)=(1,0.75,0.375,0.375) \\
B_{2}^{\prime}=A_{2}^{\prime} \circ R^{\prime}=(0,0.3525,0.71,0.26,0.0375) \\
\text { Apply the model } M(\cdot, \oplus) \text { and we obtain } \\
A_{1}^{\prime}=N\left(A_{1}\right)=(0.36,0.28,0.18,0.18) \\
B_{1}^{\prime}=A_{1}^{\prime} \circ R^{\prime}=(0,0.2,0.5484,0.2134,0.00382) \\
A_{2}^{\prime}=N\left(A_{2}\right)=(0.4,0.3,0.15,0.15) \\
B_{2}^{\prime}=A_{2}^{\prime} \circ R^{\prime}=(0,0.201,0.554,0.2105,0.0345)
\end{gathered}
$$

Now we find that the model outputs abovementioned are nearly the same as when the input vectors are basically proportional, which is consistent with people's thinking result.

Example 5 Suppose the fuzzy relation $R$ and the weight vector $A$ as follows.

$$
R=\left(\begin{array}{ccccc}
0.8 & 0.2 & 0 & 0 & 0 \\
0.9 & 0.1 & 0 & 0 & 0 \\
0.7 & 0.3 & 0 & 0 & 0 \\
0.6 & 0.3 & 0.1 & 0 & 0
\end{array}\right)
$$

and $A=(0.18,0.31,0.28,0.23)$. 
Because $R=N(R)$, then

Apply the model $M(\bigwedge, \bigvee)$ and we obtain

$$
\begin{aligned}
& A^{\prime}=S(A)=(0.58,1,0.9,0.74) \\
& B^{\prime}=A^{\prime} \circ R=(0.9,0.3,0.1,0,0)
\end{aligned}
$$

Apply the model $M(\cdot, \bigvee)$ and we obtain

$$
\begin{gathered}
A^{\prime}=S(A)=(0.58,1,0.9,0.74) \\
B^{\prime}=A^{\prime} \circ R=(0.9,0.27,0.074,0,0)
\end{gathered}
$$

Apply the model $M(\cdot, \oplus)$ and we obtain

$$
\begin{aligned}
& A^{\prime}=N(A)=(0.18,0.31,0.28,0.23) \\
& B^{\prime}=A^{\prime} \circ R=(0.757,0.22,0.023,0,0)
\end{aligned}
$$

Now we can conclude that the model outputs are nearly consistent with people's thought.

\section{Conclusion}

In this paper, we introduce two concepts of standardized fuzzy set and normalized fuzzy set, propose a novel method to improve the comprehensive evaluation model and its algorithms. Finally, we use three numerical examples to illustrate our proposed model reasonable and point out that our proposed method can avoid some inefficiencies in applications. Further, we will consider the comprehensive evaluation model for interval-number and fuzzy-number.

\section{Acknowledgment}

This work is partially supported by the National Natural Science Foundation of China (NSFC No. 10971243) and the National Natural Science Foundation of China (NSFC No. 90820010). The corresponding authors are Wenyi Zeng (zengwy@bnu.edu.cn) and Shuang Feng (fengshuang@mail.bnu.edu.cn).

And the authors would like to thank the anonymous referees for their great comments and valuable suggestions which helped us improve this paper.

\section{References}

1. H. Bustince, F. Herrera, J. Montero, "Fuzzy Sets and Their Extensions: Representation, Aggregation and Models," Heidelberg: Physica-Verlag, 2007.
2. T.E. Triantaphyllou, "Multicriteria Decisior making Methods: A Comparative Study," Kluwer Academic Publishers: Dordrecht, 2000.

3. T.L. Saaty, "The Analytic Hierarchy Process," McGraw-Hill: New York, 1980.

4. L.A. Zadeh, "Fuzzy sets," Inform. Control, 8, 338353(1965).

5. R.E. Bellman, L.A. Zadeh, "Decision-making in a fuzzy environment," Management Sciences, 17, 141164 (1970).

6. D. Dubois, H. Prade, "Fuzzy sets and systems: theory and applications," Academic Press: New York, 1980.

7. A. Kaufmann, M.M. Gupta, "Introduction to fuzzy arithmetic theory and applications," Van Nostrand Reinhold: New York, 1991.

8. H.X. Li, V.C. Yen, "Fuzzy sets and fuzzy decisionmaking," CRC Press: New York, 1995.

9. P.Z. Wang, "Fuzzy set theory and its applications," Shanghai Science and Technology Press: Shanghai, 1984. (in chinese)

10. S. Feng, L.D. Xu, "Decision support for fuzzy comprehensive evaluation of urban development," Fuzzy Sets and Systems, 105, 1-12(1999).

11. Z.H. Zou, Y. Yi, J.N. Sun, "Entropy method for determination of weight of evaluating indicators in fuzzy synthetic evaluation for water quality assessment," Journal of Environmental Sciences, 18, 10201023(2006).

12. Y.Y. Chen, W.Q. Zhang, "Comprehensive decisionmaking and weather forecast," Mathematics in Practice and Theory, 113, 32-39 (2000). (in chinese)

13. Y. Qiao, X. Liu, L. Zou, "Evaluating the quality of education via linguistic aggregation operator," ICIC Express Letters, 4, 1851-1856(2010).

14. Y.F. Kuo, P.C. Chen, "Selection of mobile value-added services for system operators using fuzzy synthetic evaluation," Expert Systems with Applications, 30, 612-620(2006).

15. D. Xia, B. Chen, "A comprehensive decision-making model for risk management of supply chain," Expert Systems with Applications, 38, 4957-4966(2011).

16. Z.H. Liang, K. Yang, Y.W. Sun, J.H. Yuan, H.W. Zhang, Z.Z. Zhang, "Decision support for choice optimal power generation projects: fuzzy comprehensive evaluation model based on the electricity market," $\mathrm{En}$ ergy Policy, 34, 3359-3364(2006).

17. T. Samatsu, K. Tachikawa, Y. Shi, "Usability improvement for a car retrieval system employing the important degrees of fuzzy grades," International Journal of Innovative Computing, Information and Control, 5, 5061-5068(2009).

18. Y.J. Guo, Y. Yao, P.T. Yi, "Method and Application of dynamic Comprehensive Evaluation," Systems Engineering Theory and Practice, 27, 154-158(2007). (in chinese) 